\title{
Soldadura de metales disimilares por láser de $\mathrm{CO}_{2}$
}

\author{
F. Garciandía ${ }^{(*)}$, F. Zubiri $^{(*)}$, J.L. Etayo ${ }^{(*)}$, R. Cervantes ${ }^{(*)}$ e I. Iriberri ${ }^{(*)}$
}

Resumen En el presente documento se resumen los trabajos llevados a cabo en CETENASA (Dpto. Láser) para la obtención de puntos de soldadura entre metales disimilares. El par metálico estudiado es M-35 y F-143, correspondientes a un acero rápido y un acero para muelles, respectivamente. Debido a las composiciones químicas de las aleaciones implicadas se comprende la dificultad para obtener soldaduras con estructuras aceptables, desde el punto de vista metalúrgico, dado el alto grado de fisuración que presentan estos materiales, especialmente el M-35. El presente trabajo es un avance de los primeros resultados encontrados dentro de una investigación más amplia, en la que se demuestran las posibilidades que presenta la soldadura con láser de $\mathrm{CO}_{2}$.

Palabras clave: Soldadura. Láser $\mathrm{CO}_{2}$. Metales disimilares.

\section{Welding of dissimilar metals by $\mathrm{CO}_{2}$ lasers}

\begin{abstract}
The work carried out in CETENASA (Laser department) in order to weld dissimilar metals is summarized. The involved metallic pair is M-35 and F-143, a high speed steel and a spring steel, respectively. Looking at the chemical composition of the involved alloys that will appear later, it can be easily understood the difficulty to obtain welded parts with structures metallurgicaly acceptable because of the high cracking degree that these materials show, specially M-35. The principles of a study which is being developed in the authors' laboratory and which shows some interesting $\mathrm{CO}_{2}$ laser possibilities are presented.
\end{abstract}

Keywords: Welding. Laser $\mathrm{CO}_{2}$. Dissimilar metals,

\section{INTRODUCCIÓN}

Las ventajas que presenta el láser en muchas aplicaciones de soldadura, así como la experiencia positiva sobre la soldadura de aleaciones disimilares, se resumen en las líneas siguientes, en un trabajo que responde a una necesidad industrial concreta. La posibilidad de tener en una misma pieza las características diferentes de dos materiales distintos según sus funciones en dicha pieza da una idea de las repercusiones técnico-económicas que ello conlleva (1 y 2).

\section{DESCRIPCIÓN EXPERIMENTAL}

Los ensayos se han realizado sobre las aleaciones M-35 y F-143, cuya composición es la siguiente: M-35: 0,9\% C, $6 \% \mathrm{~W}, 5 \% \mathrm{Mo}, 2 \% \mathrm{~V}, 5 \% \mathrm{Co}$,

(*) CETENASA; Polígono de Elorz s/n. 31110-Pamplona (España).
$4 \%$ Cr y F-143: 0,5 \% C, 0,15\% V, 0,25\% Si, $1 \%$ $\mathrm{Cr}, 0,85 \% \mathrm{Mn}$. En ambos casos, el material se encuentra en estado de templado y revenido.

Las pruebas de soldadura se realizaron con la asistencia de dos generadores láser $\mathrm{CO}_{2}$ de diferente diseño, tecnología y potencia. Por un lado, se utilizó un láser de $\mathrm{CO}_{2}$ de flujo transversal lento de 5 $\mathrm{kW}$ y, por otro, se realizaron pruebas con un láser de $\mathrm{CO}_{2}$ de flujo axial rápido de $800 \mathrm{~W}$ de potencia.

Se fijaron unas premisas de partida, en parte debidas a las necesidades industriales por las que se habían elegido las aleaciones anteriores. Una de ellas era la profundidad de penetración de la soldadura que quedó fijada en $1 \mathrm{~mm}$.

Inicialmente se hicieron pruebas con ambos láseres utilizando parámetros convencionales de soldadura. El resultado, en todos los casos, fue el esperado: se obtenía un punto o un cordón absolutamente fisurado desde la raíz a la superficie.

Esto hizo que se replanteara toda la situación y, en este sentido, se trabajó en dos líneas diferentes que a continuación se tratarán de explicar. 


\section{RESULTADOS Y DISCUSIÓN}

\subsection{Soldadura del par con pre y post- calentamiento a partir de una fuente térmica diferente al láser}

La necesidad de evitar la fisuración evidente que se producía al aplicar el láser directamente hizo pensar en la posibilidad de utilizar otro medio diferente al láser que, por medio de un aporte térmico, realizara una función de precalentamiento y postcalentamiento antes y después de ejecutar la soldadura láser propiamente dicha.

Para ello se preparó el montaje que aparece en la figura 1, donde lo que básicamente se tiene es una placa térmica que precalienta las probetas a soldar hasta una temperatura de $350{ }^{\circ} \mathrm{C}$. Para controlar las temperaturas obtenidas se disponía de un termopar, que, a través de un aparato de medida, informaba en todo momento de la temperatura en la parte inferior de las probetas a soldar, así como en la parte superior de las mismas. El equilibrio térmico, de todas maneras, se alcanzaba con cierta rapidez dado el tamaño $(25 \times 10 \mathrm{~mm}$.) y espesor (aprox. $3 \mathrm{~mm}$.) de las piezas.

En estos experimentos, la fuente láser empleada fue un $\mathrm{CO}_{2}$ de flujo axial rápido, de $800 \mathrm{~W}$, pero que, en el momento de su utilización, suministraba una potencia máxima de salida de $700 \mathrm{~W}$. Para una mejor absorción de la radiación láser, dada la alta reflectividad de los metales en general y considerando lo limitado de la potencia empleada, se pintaron las probetas con pintura negra mate anticalórica (que soporta hasta $600-800{ }^{\circ} \mathrm{C}$ ). $\mathrm{El}$ gas de aporte utilizado fue argón.

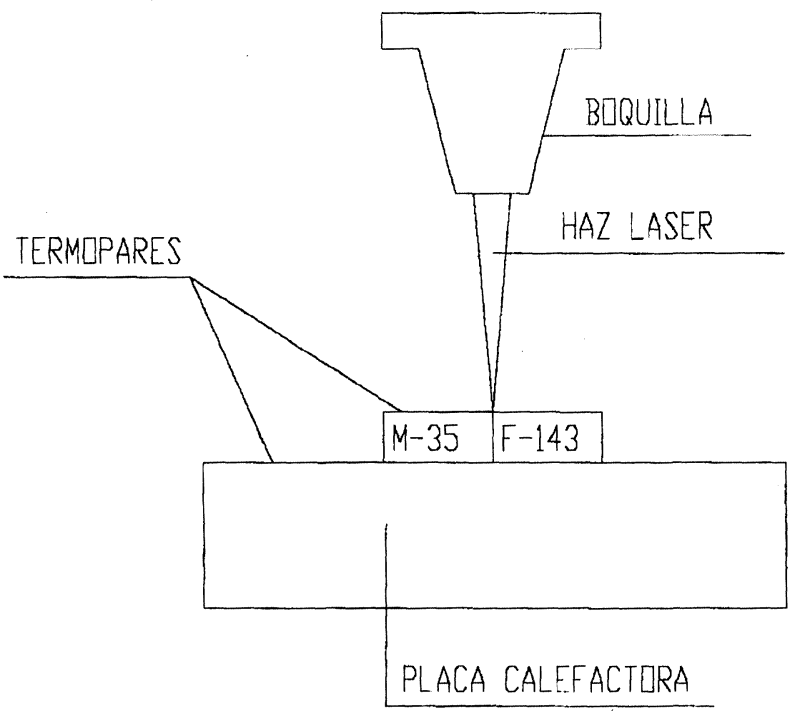

Fir. 1.- Montaje experimental para soldadura del par con precalentamiento externo.

FIG. 1.- Experimental arrangement for laser welding with external heating.
Para la potencia mencionada de $700 \mathrm{~W}$ de salida, el tiempo de interacción del láser era de aproximadamente 0,5 s. A partir de la ejecución, se paraba el suministro de calor de la placa calórica, disminuyendo lentamente la temperatura desde los $300-400{ }^{\circ} \mathrm{C}$ hasta la temperatura ambiente. Se procedió de esta manera para la soldadura del par M35-F-143. También se realizó un cordón de soldadura de unos $20 \mathrm{~mm}$. de longitud entre dos probetas de M-35 (lo cual, si cabe, es más desfavorable desde el punto de vista de la fisuración) a una velocidad de $1 \mathrm{~m} / \mathrm{min}$ obteniéndose en ambos casos buenos resultados.

El análisis microestructural de los dos casos, se resume a continuación:

\subsubsection{Sección del cordón M-35-M35}

La microestructura del material está formada por martensita revenida y carburos uniformemente distribuidos en la matriz del material, siendo el estado del material de partida de temple y revenido. En la zona afectada térmicamente (ZAT), se observa un ligero engrosamiento del tamaño de grano austenítico, así como cierta disolución de carburos, tanto más acusada cuanto mayor es la proximidad al material fundido durante el proceso de soldadura. En la la figura 2 se muestra la ZAT, forma y penetración del cordón de soldadura. La microestructura del cordón de soldadura está formada por martensita y carburos, adoptando una morfología dendrítica generada durante la fase de solidificación del material.

\subsubsection{Sección del punto de soldadura entre M-35 y F-143}

Del análisis metalográfico, se desprende que la microestructura del acero F-143 está formada por colonias de martensita revenida fina, junto con estructuras de tipo bainítico y perlita fina. La ZAT del material está formada por agujas de martensita.

Para el' acero M-35, la microestructura está formada por martensita revenida con carburos uniformemente distribuidos en la matriz del material. En la figura 3 se muestra la forma y penetración del punto de soldadura, observándose la presencia de algunos poros en la línea de fusión del acero rápido M-35.

\subsubsection{Ensayo de dureza}

Se han realizado barridos de dureza en las zonas de soldadura y ZAT. El barrido de durezas se ha realizado por el método Vickers con carga de $0,5 \mathrm{~kg}$ y 15 s de aplicación de la carga. Los resultados se muestran a continuación: 


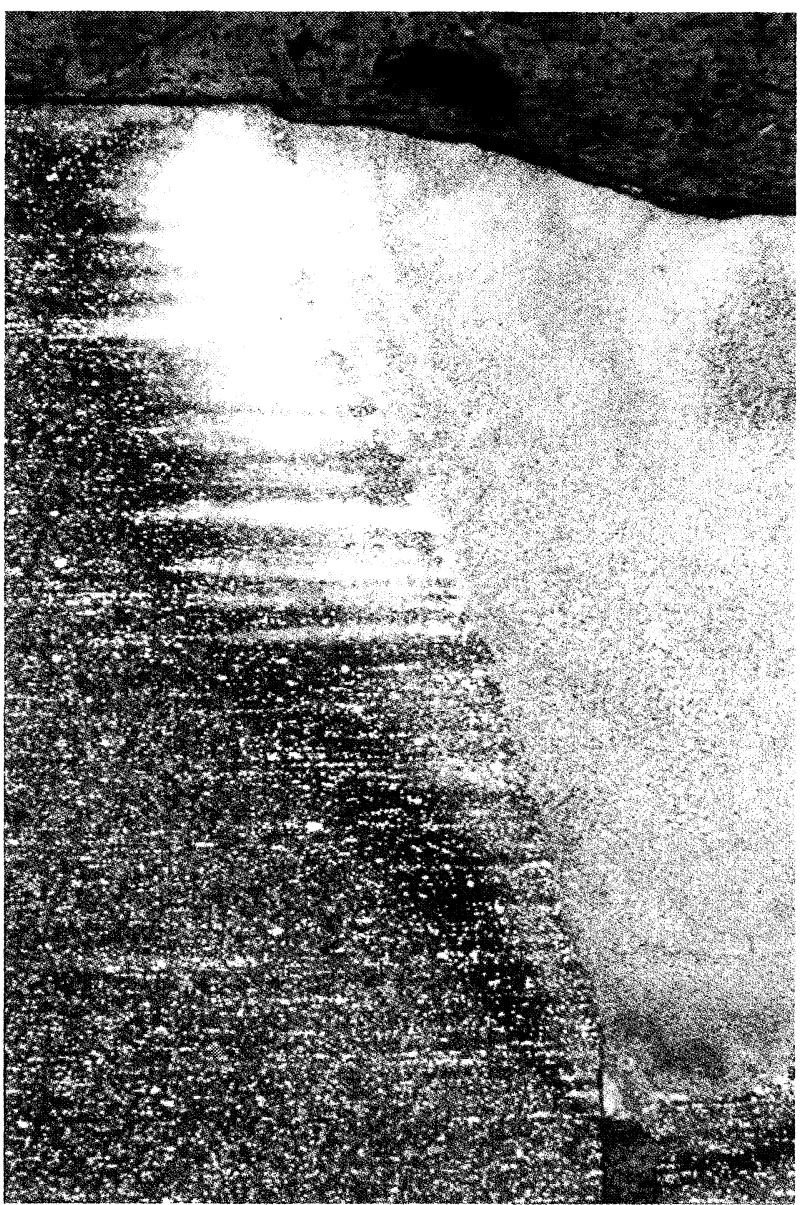

FIG. 2.- Micrografía de la soldadura del par M-35M35.

FIG. 2.- Micrograph of laser welding of M35-M35.

Soldadura M35-M35

Dureza del material base:

912 - $926 \mathrm{HV} / 0,5$

Dureza ZAT:

$965-997 \mathrm{HV} / 0,5$

Dureza cordón de soldadura: $\quad 797$ - 812 HV/0,5

Soldadura M-35 - F-14

Dureza material base F-143:

420 - $440 \mathrm{HV} / 0,5$

Dureza material base M-35:

Dureza ZAT F-143:

$906-915 \mathrm{HV} / 0,5$

$736-740 \mathrm{HV} / 0,5$

945 - $965 \mathrm{HV} / 0,5$

Dureza ZAT M-35:

$725(\mathrm{~F}-143)-$

812(M-35) HV/0,5

\subsection{Soldadura del par con pre y post- calentamiento utilizando el propio láser}

Una vez superada la fase anterior, se dio un paso más con la idea de sustituir, la placa térmica utilizada en el punto anterior para el pre-postcalentamiento, por el propio láser, de manera que, por medio de

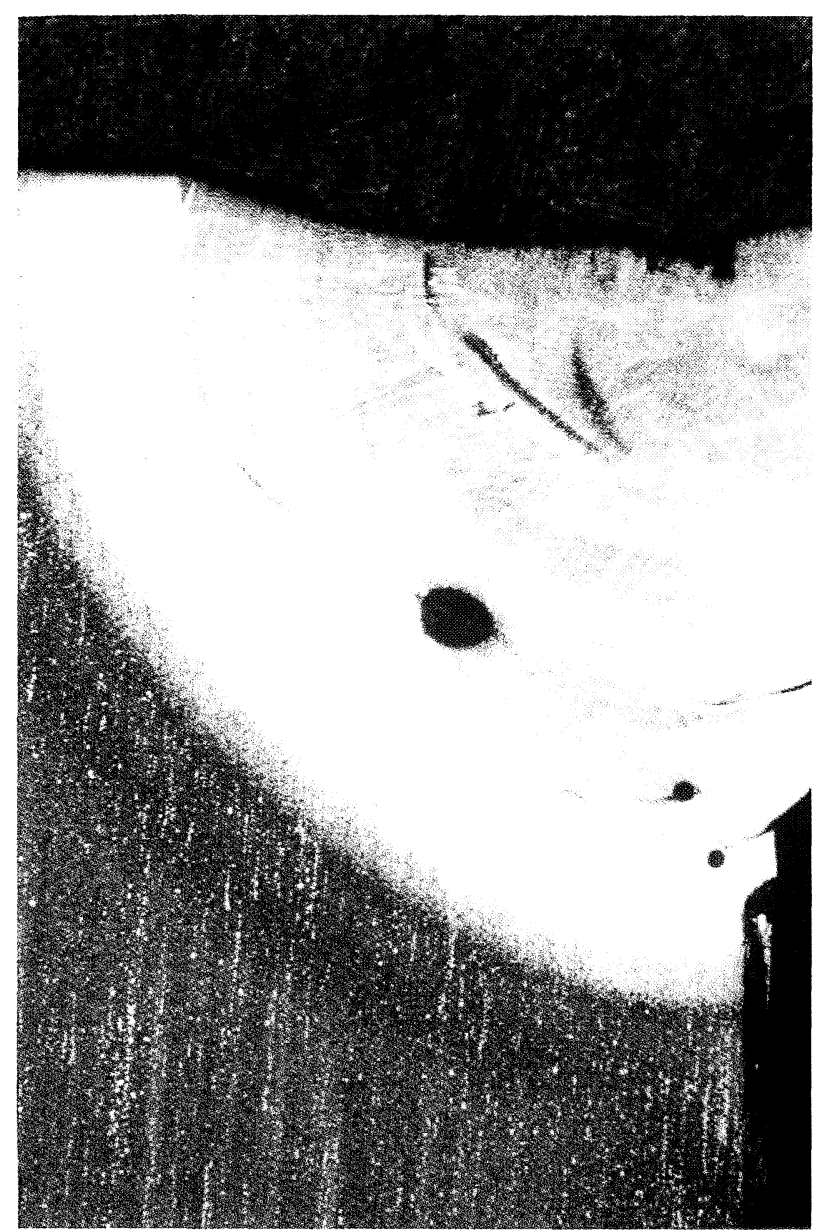

FIG. 3.- Detalle del cordón del par M35-F143.

FIG. 3.-Detail of M35-F143 track.

la variación de potencia en el generador y por medio del movimiento del eje en que se sujeta el cabezal de soldadura, conseguir una desfocalización $\mathrm{y}$, por tanto, una densidad de potencia inferior a la que se obtiene en la soldadura.

El fundamento del experimento se puede observar en la figura 4. Las probetas se pintaron de la forma descrita en el punto anterior.

Lógicamente el campo que se puede abarcar con estos parámetros es muy grande y se hace necesario partir de unos ciertos valores o moverse en una zona discreta de trabajo, en parte debido a cuestiones prácticas de aplicación industrial posterior. Es decir, el tiempo real para conseguir una soldadura debe ser "razonablemente" corto, porque si no puede ser ya de entrada poco interesante desde el punto de vista económico.

Por ello, se desarrolló un cálculo por medio de elementos finitos (cuyos pormenores no son el objeto del presente documento) con el fin de acotar precisamente el campo de trabajo. Así, para la penetración dada de $1 \mathrm{~mm}$, se obtenían valores que hacían pensar que se debería trabajar en alturas de 


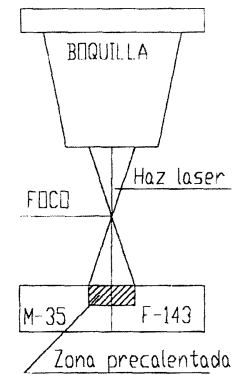

$(0)$

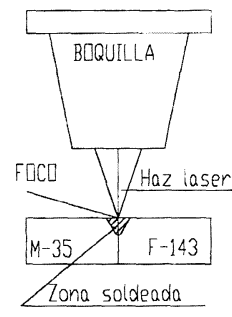

(b)

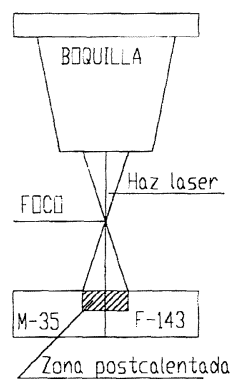

$(c)$
FIG. 4.- Montaje experimental con las etapas del calentamiento láser.

FIG. 4.- Experiemental arrangement with laser heating stages.

desfocalización (por encima del punto focal) de 10 a $50 \mathrm{~mm}$ con tiempos de interacción que podían ir desde milisegundos hasta varios segundos y con potencias que fluctuasen entre 150 y $700 \mathrm{~W}$, en el caso del láser $\mathrm{CO}_{2}$ de $800 \mathrm{~W}$, y una potencia máxima de unos $1.500 \mathrm{~W}$ era la recomendada para el láser de $5 \mathrm{~kW}$.

La temperatura se controlaba por medio del termopar mencionado en el apartado anterior, pero lógicamente esta medida se debía de tomar con muchas reservas, dado que el termopar, en el mejor de los casos, se situaba $1 \mathrm{~mm}$ por debajo de la raíz del futuro punto de soldadura y, por supuesto, en las interacciones rápidas de milisegundos no le daba tiempo a reaccionar.

De todos modos, los mejores resultados se obtuvieron utilizando el láser de menos potencia con una potencia de pre y post-calentamiento de aproximadamente $150 \mathrm{~W}$, con tiempos muy cortos de interacción (del orden de $200 \mathrm{~ms}$ ) y con un tiempo de interacción para la soldadura a $750 \mathrm{~W}$ de $500 \mathrm{~ms}$. Las velocidades de posicionamiento del cabezal desde la altura de precalentamiento hasta el punto focal de soldadura y desde éste hasta la de postcalentamiento fueron rápidas (del orden de 3 a 5 $\mathrm{m} / \mathrm{min}$.). En dichos cordones, se apreciaban pequeñas grietas y algunos poros, pero, sin duda, se observa una gran mejoría, si lo comparamos con una macrografía de un punto de soldadura láser convencional que presentaban una grieta total desde la raíz hasta la cabeza del cordón.

Los ensayos de dureza a que se sometieron las mejores probetas soldadas arrojaron los siguientes resultados:

$\begin{array}{ll}\text { ZAT F-143 } & 650-750 \mathrm{HV} / 0,5 \\ \text { ZAT M-35 } & 800-900 \mathrm{HV} / 0,5 \\ \text { CORDON } & 750-850 \mathrm{HV} / 0,5\end{array}$

\section{CONCLUSIONES}

A lo largo del trabajo que se ha intentado resumir en el presente documento se ha constatado la dificultad existente a la hora de soldar los metales mencionados (M-35 y F-143) entre sí. No obstante, a través de las pruebas llevadas a cabo se constata un esperanzador avance en algo que prácticamente está en sus inicios y que de arrojar resultados positivos puede ofrecer soluciones a problemas industriales que hoy en día no son abordables.

Quedan todavía muchas dudas y problemas que solucionar para mejorar los resultados obtenidos en el punto 3. Téngase en cuenta que cuando se habla de interacciones de milisegundos son éstos tiempos programados en un CNC y se debe tener en cuenta también que las transiciones de las líneas de programa, aceleraciones y deceleraciones del cabezal, tiempos de respuesta del láser, etc. son del mismo orden, con lo cual se genera un error que no puede ser despreciado. Asimismo, se espera mejorar las condiciones y métodos de control de la temperatura para poder incidir en los parámetros de manera más eficaz.

\section{REFERENCIAS}

(1) SÄNDIG, S. S. Technological Aspects of Laser Joining of Steel and Hard Metal Components; ICALEO'95. Proc. LIA 80, 1995: 895-902.

(2) Cruciani, D. y Linguiti, F. Laser welding dissimilar metals as alternative to brazing: Experimental results, economical and quality evaluations, applications in production line; $\mathrm{CO}_{2}$ Lasers and Applications II; SPIE. 1276, 1990: 243-246. 